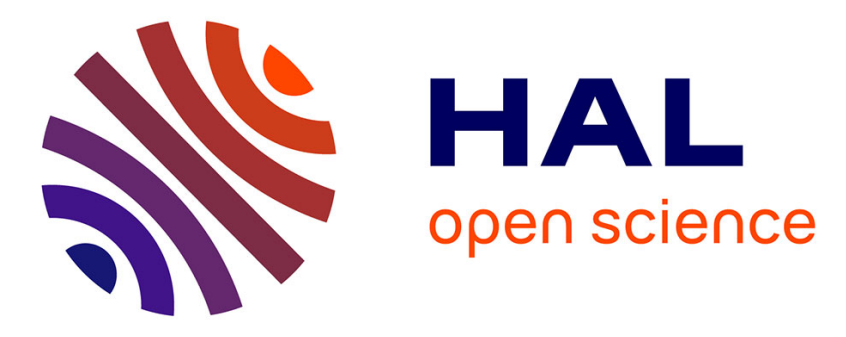

\title{
Discrete Controllability of Distributed Parameters Systems using Lattice Boltzmann Models: an application to the Shallow Water Equations
}

Diemer Anda Ondo, Laurent Lefèvre, Bastien Chopard

\section{- To cite this version:}

Diemer Anda Ondo, Laurent Lefèvre, Bastien Chopard. Discrete Controllability of Distributed Parameters Systems using Lattice Boltzmann Models: an application to the Shallow Water Equations. IFAC World Congress, Aug 2011, Milan, Italy. pp.9206-9211, 10.3182/20110828-6-IT-1002.02546 . hal-00688639

\section{HAL Id: hal-00688639 \\ https://hal.science/hal-00688639}

Submitted on 18 Apr 2012

HAL is a multi-disciplinary open access archive for the deposit and dissemination of scientific research documents, whether they are published or not. The documents may come from teaching and research institutions in France or abroad, or from public or private research centers.
L'archive ouverte pluridisciplinaire HAL, est destinée au dépôt et à la diffusion de documents scientifiques de niveau recherche, publiés ou non, émanant des établissements d'enseignement et de recherche français ou étrangers, des laboratoires publics ou privés. 


\title{
Discrete Controllability of Distributed Parameters Systems using Lattice Boltzmann Models: an application to the Shallow Water Equations
}

\author{
Diemer Anda Ondo* Laurent Lefèvre ${ }^{* *}$ Bastien Chopard ${ }^{* * *}$ \\ * LCIS, 50 rue Barthélémy de Laffémas, BP54 26902 VALENCE \\ Cedex 09, France \\ ** LAGEP, Université Claude Bernard Lyon 1, bât 308G \\ ESCPE-Lyon, 43 bd du 11 Novembre 1918, 69622 Villeurbanne Cedex, \\ France \\ *** University of Geneva, Batelle Building A, 7 route de Drize, 1227 \\ Carouge, Switzerland
}

\begin{abstract}
The Lattice Boltzmann (cellular automata) model equivalent to the 1D shallow water equations is first introduced. Then, its state-space representation with the introduction of input-output variables is presented. Finally, the discrete controllability problem is investigated. First considerations and methods suitable for large scale discrete dynamical systems Gramian computations are presented. Then controllability analysis arguments are developed based on the obtained Gramians and on the convergence of energy properties.
\end{abstract}

Keywords: Lattice Boltzmann models, controllability and observability gramians, Lyapunov equations, distributed parameters systems, Shallow water equations.

\section{INTRODUCTION}

Discrete models are extensively used to describe simply complex distributed parameters systems. These discrete models are often obtained from the discretization of partial differential equations. However, more recently, models have been built to represent dynamical systems directly from discrete formulations of quantitative behaviors or physical principles. Cellular automata models are examples of such an approach. Historically they have been introduced by von Neuman and Ulam Neuman (1966) as self-reproducing automata. However, more recently, they have been extensively used to model distributed parameters systems in epidemiology Ostfeld et al. (2005); Slimi et al. (2009), ecosystems and population spatial dynamics Chopard and Lagrava (2006); Hencinas et al. (2007), biomedical applications Ouared et al. (2006); Chopard et al. (2010), etc. In these application examples discrete state space are convenient. However in many distributed parameters systems based on conservation laws for macroscopic variables (in the thermodynamical meaning), continuous state spaces seem more appropriate. For this reason, Lattice Boltzmann Models (LBM) are a convenient extension of cellular automata models as they have realvalued states. LBM have been used also to represent various kinds of physical models Chopard and Droz (2005). They are now recognized as a powerful way to solve NavierStokes equations. In this paper, we will first introduce them in a general framework and then consider a particular application to fluid flow modeling and control. LBM have been recently developed for $1 \mathrm{D}$ and $2 \mathrm{D}$ shallow water flows Pham et al. (2010); Zhou (2004); Salmon (1999). They have proved to be very efficient alternative solutions to classical discretizations of Shallow Water equations Pham et al. (2010), especially to describe large and complex free surface water transportation systems. However, very little is known about their behavior in what concern qualitative dynamical properties. This paper is an attempt to present LBM in a state space formalism suitable for the analysis of these properties. In section 2, the LBM for shallow water equation is established. In section 3, the Gramian computation problem is developed. Finally, in section 4, the obtained results for the Gramians are analyzed

\section{FROM THE LOCAL LATTICE-BOLTZMANN MODEL TO THE GLOBAL STATE SPACE REPRESENTATION}

We will consider in the following the example of water flow in a regular open channel with slope $I$ and width $B$, as represented in Fig.1. Under the shallow water assumptions Pham et al. (2010), the water levels and flow in such a canal reach satisfy:

$$
\begin{aligned}
& \partial_{t} h+\partial_{x}(h u)=0 \\
& \partial_{t}(h u)+\partial_{x}\left(\frac{1}{2} g h^{2}+h u^{2}\right)=F
\end{aligned}
$$

where $h$ denotes the water depth, $u$ the depth-averaged horizontal velocity of the flow and $g$ the gravitational acceleration. The force term, $F=g h(I-J)$, accounts for the bed slope, $I$, and the bed friction, $J$, which is modelled with the classical Manning formula: $J=$ $n^{2} u^{2} /\left(\frac{B h}{B+2 h}\right)^{4 / 3}$, with $n$ the Manning's coefficient. 


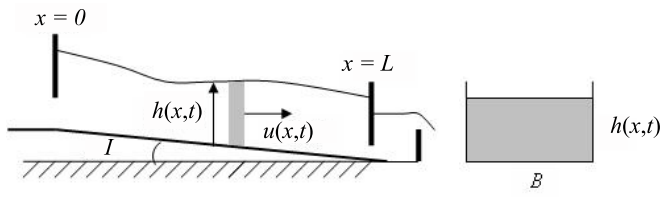

Fig. 1. Longitudinal (left) and lateral (right) views of an open rectangular channel

\subsection{The Lattice Boltzmann Model}

The Lattice Boltzmann (LB) method has proven to be a powerful numerical tool to simulate the fluid flows and many other physical phenomena in a fully discrete (time and space) grid Chopard and Droz (2005). The dynamics in LBM consists of an alternation of collision and streaming phases:

$$
\begin{array}{ll}
f_{i}^{\text {out }}(x, t)=f_{i}^{\text {in }}(x, t)+\Omega_{i}(f(x, t)) & \text { Collision } \\
f_{i}^{\text {in }}\left(x+v_{i} \delta t, t+\delta t\right)=f_{i}^{\text {out }}(x, t) & \text { Streaming }
\end{array}
$$

where $f^{i n}$ denotes the vector of all density distributions, $f_{i}^{i n}$, of particles entering a site, $f_{i}^{\text {out }}$ denotes those distributions which result from the collision, $\delta x$ is the lattice spacing and $\delta t$ is the time step. $\Omega_{i}$ is the collision operator, which is commonly defined by the Bhatnagar-Gross-Krook (BGK) model Bhatnagar et al. (1954): $\Omega_{i}=\frac{1}{\tau}\left(f_{i}^{e q}-f_{i}\right)$, where $\tau$ is a relaxation time and $f_{i}^{e q}$ are the equilibrium distribution functions which depend on the physical process to be described. By combining the equations in (2), we obtain the evolution equation

$$
f_{i}\left(x+v_{i} \delta t, t+\delta t\right)=f_{i}(x, t)+\frac{1}{\tau}\left(f_{i}^{e q}-f_{i}\right)
$$

where $f$ stands for $f^{\text {in }}$ and where the spatial and temporal arguments of the term $f_{i}^{e q}-f_{i}$ is the same as the first term of the right hand side. Here we will consider a $1 \mathrm{D}$ lattice termed $D 1 Q 3$ (1D spatial grid with densities or velocities in each point, see Fig. 2) with a lattice spatial spacing $\delta x$ and a time step $\delta t$. This results in a lattice (or numerical) velocity $v=\frac{\delta x}{\delta t}$. Thus we assign for the three velocities in (Fig. 2) the values $v_{0}=0, v_{1}=v$ and $v_{2}=-v$. In

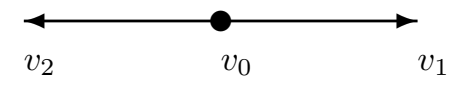

Fig. 2. Lattice D1Q3

the Shallow Water example, external forces have to be considered in the evolution equations. For a constant force, this can be done by adding the force terms $F$ in equations (3) in the following way Chopard and Droz (2005):

$$
f_{i}(x+v \delta t, t+\delta t)=f_{i}(x, t)+\frac{1}{\tau}\left(f_{i}^{e q}-f_{i}\right)+w_{i} \frac{\delta t}{c_{s}^{2}} v_{i} F(4)
$$

Many parameters and unknown equilibrium distributions appear in such a LBM. Usually, they all are determined by the physical assumptions required for the model. For instance, in the D1Q3 case, symmetry reasons lead to a choice of the parameters $w_{i}$ and $c_{s}^{2}$ which must satisfy Zhou (2004)

$$
\begin{aligned}
& \sum_{i} w_{i}=1, \quad \sum_{i} v_{i} w_{i}=0, \quad \sum_{i} v_{i}^{2} w_{i}=c_{s}^{2}, \\
& \sum_{i} v_{i}^{3} w_{i}=0, \quad \sum_{i} v_{i}^{4} w_{i}=3 c_{s}^{4}
\end{aligned}
$$

This implies $w_{0}=\frac{2}{3}, \quad w_{1}=w_{2}=\frac{1}{6}, \quad c_{s}^{2}=\frac{v^{2}}{3}$. On another hand the equilibrium distributions may be chosen in order to give rise to the "correct" (or physical) balance equations Chopard and Droz (2005). In the 1D Shallow Water example, this lead to the following relations between microscopic (or mesoscopic) values of the equilibrium distributions and the three "macroscopic variables" which are to total mass, momentum and energy. The three balance equations read in this case Zhou (2004):

$$
\begin{array}{cl}
\sum_{i} f_{i}^{e q}=h, & \sum_{i} v_{i} f_{i}^{e q}=h u \\
\sum_{i} v_{i}^{2} f_{i}^{e q}= & \frac{1}{2} g h^{2}+h u^{2}
\end{array}
$$

They lead to

$$
\begin{aligned}
f_{0}^{e q} & =h-\frac{1}{2 v^{2}} g h^{2}-\frac{1}{v^{2}} h u^{2} \\
f_{1}^{e q} & =\frac{1}{4 v^{2}} g h^{2}+\frac{1}{2 v} h u+\frac{1}{2 v^{2}} h u^{2} \\
f_{2}^{e q} & =\frac{1}{4 v^{2}} g h^{2}-\frac{1}{2 v} h u+\frac{1}{2 v^{2}} h u^{2}
\end{aligned}
$$

Finally, the macroscopic properties $h$ and $u$ must satisfy at any time the relations

$$
\begin{aligned}
h & =\sum_{i} f_{i}=f_{0}+f_{1}+f_{2} \\
h u & =\sum_{i} v_{i} f_{i}=v f_{1}-v f_{2}
\end{aligned}
$$

The variables $h$ and $u$, are the variables classically used to describe Shallow Water flows.

\subsection{Linearized state space evolution equation}

The state space in the case of a LBM is the set of values for the distribution functions vector. It depends of dimension and kind of lattice (see Chopard and Droz (2005); Zhou (2004)). In a lattice with $n$ sites where each site has $|v|$ neighbors, the state space is $Z_{l b m}=\left(\left(\mathbf{R}^{+}\right)^{|v| n}\right)$.

Local dynamic: The local dynamic is defined by the distribution functions related to "particles" arriving at time $t+1$ on a given site from the neighbor sites where they were at time $t$. In our $D 1 Q 3$ lattice case, after normalizing the time and space steps, these dynamics read:

$$
f_{i}\left(x_{k}, t+1\right)=f_{i}\left(x_{k+\bar{v}}, t\right)+\frac{1}{\tau}\left(f_{i}^{e q}-f_{i}\right)
$$

with $i=0,1,2$ and $\bar{v}=\frac{v_{i}}{v}$.

Global dynamic: The global dynamic is obtained by closing the set of local dynamics equations (9) with the closure relations (7) for the distributions equilibrium profiles. In our D1Q3 lattice case, these nonlinear dynamics read:

$$
f_{i}\left(x_{k}, t+1\right)=A_{i}\left[\begin{array}{l}
f_{0}\left(x_{k+\bar{v}}, t\right) \\
f_{1}\left(x_{k+\bar{v}}, t\right) \\
f_{2}\left(x_{k+\bar{v}}, t\right)
\end{array}\right]
$$


with $i=0,1,2$. Defining the state space vector as

$$
\begin{aligned}
& x(k)=\left[f_{0}\left(x_{1}, k\right) f_{1}\left(x_{1}, k\right) f_{2}\left(x_{1}, k\right) \quad \ldots\right. \\
& \left.f_{0}\left(x_{n}, k\right) f_{1}\left(x_{n}, k\right) f_{2}\left(x_{n}, k\right)\right]^{T},
\end{aligned}
$$

we obtain a classical evolution model of the form

$$
x(k+1)=A(F r, \Psi) x(k)
$$

with the matrices $A_{i}, i=0,1,2$, and $A$ given in the appendix. It is remarkable that these matrices only depend on the flow Froude number, $F r^{2}=\frac{u^{2}}{g h}$, and the lattice Froude number, $\Psi^{2}=\frac{v^{2}}{g h}$. These two dimensionless numbers characterize the kind of flow which arises locally at the considered location (see appendix A). It is important to notice that, since $F r$ and $\Psi$ are functions of $h$ and $u$ which are in turn functions of the distributions $f_{i}$ (see equations (8)), we have $A=A(x)$ and the global evolution equation (12) is nonlinear.

Linearized global dynamics: We will consider the linearization around stationary equilibrium profiles for the water levels $h_{e}$ and flows $h_{e} u_{e}$ in the classical sense. Note that in general, equilibrium profiles for the water levels $\left(h_{e}\right)$ or depth-averaged horizontal velocities $\left(u_{e}\right)$ in the Saint-Venant equations (1) need not to be uniform stationary profiles. These equilibrium profiles correspond to distribution functions $\bar{f}_{i}$ such that $\sum_{i} \bar{f}_{i}=h_{e}$ and $\sum_{i} v_{i} \bar{f}_{i}=h_{e} u_{e}$. Note also that these stationary distributions are not the equilibrium distributions, $f_{i}^{e q}$ in the Maxwell Boltzmann relaxation meaning which are given in equations (7). Writing $f_{i}=\bar{f}_{i}+\epsilon_{i}$, one obtains for the macroscopic variables:

$$
\begin{aligned}
h & =\sum_{i} f_{i}=h_{e}+\sum_{i} \epsilon_{i} \\
h u & =\sum_{i} v_{i} f_{i}=h_{e} u_{e}+v\left(\epsilon_{1}-\epsilon_{2}\right) \\
u & =u_{e}-\frac{u_{e}}{h_{e}} \sum_{i} \epsilon_{i}+\frac{v}{h_{e}}\left(\epsilon_{1}-\epsilon_{2}\right) \\
h u^{2} & =h_{e} u_{e}^{2}-u_{e}^{2} \sum_{i} \epsilon_{i}+2 v u_{e}\left(\epsilon_{1}-\epsilon_{2}\right)
\end{aligned}
$$

The LBM evolution equation (3) leads to the evolution equation for the variations $\epsilon_{i}$

$$
\begin{aligned}
\epsilon_{i}\left(x_{k}+v_{i} \delta t, t+\delta t\right)= & \left(1-\frac{1}{\tau}\right) \epsilon_{i}\left(x_{k}, t\right)+ \\
& \frac{1}{\tau}\left(f_{i}^{e q}-\bar{f}_{i}\right)
\end{aligned}
$$

Since the distribution functions $f^{e q}$ and $\bar{f}_{i}$ are related, one gets the evolution equations on the linearized variables (variations $\epsilon_{i}$ ):

$$
\epsilon_{i}\left(x_{k}, t+1\right)=T_{i}\left[\begin{array}{c}
\epsilon_{0}\left(x_{k+\bar{v}}, t\right) \\
\epsilon_{1}\left(x_{k+\bar{v}}, t\right) \\
\epsilon_{2}\left(x_{k+\bar{v}}, t\right)
\end{array}\right], \quad i=0,1,2
$$

Defining similarly to previously the linearized state as

$$
\begin{aligned}
& z(k)=\left[\epsilon_{0}\left(x_{1}, k\right) \epsilon_{1}\left(x_{1}, k\right) \epsilon_{2}\left(x_{1}, k\right) \quad \ldots\right. \\
& \left.\epsilon_{0}\left(x_{n}, k\right) \epsilon_{1}\left(x_{n}, k\right) \epsilon_{2}\left(x_{n}, k\right)\right]^{T},
\end{aligned}
$$

we obtain around the stationary profiles $\left(h_{e}, u_{e}\right)$ a linearized autonomous state dynamical system

$$
z(k+1)=T z(k)
$$

with the matrices $T_{0}, T_{1}, T_{2}$ and $T$ given in the appendix.

Adding the force terms: The force term $F$ may be linearized around $\left(h_{e}, u_{e}\right)$ as well by neglecting the terms of order two and higher in power of $\epsilon_{i}$ series expansion. For a site $x_{k}$, one obtains:

$$
\epsilon_{i}\left(x_{k}, t+1\right)=\left(T_{i}+\bar{v} N\right)\left[\begin{array}{l}
\epsilon_{0}\left(x_{k+\bar{v}}, t\right) \\
\epsilon_{1}\left(x_{k+\bar{v}}, t\right) \\
\epsilon_{2}\left(x_{k+\bar{v}}, t\right)
\end{array}\right]+\bar{v} g h_{e}\left(I-J_{e}\right)
$$

where $J_{e}=J\left(h_{e}, u_{e}\right)$, and $N$ is given in the appendix. Then the linearized LBM with force terms may be written:

$$
z(k+1)=T_{f} z(k)
$$

where the state $z(k)$ is defined as before (see (16)) and with $T_{f}$ given in appendix.

\subsection{Inputs and outputs}

From a control point of view, once a global state space model has been obtained from the LBM, inputs and outputs variables need still to be defined. In this paper we will focus on the input variables denoted hereafter $\mathbf{u}$ (to make the difference with the macroscopic velocity $u$ clear). We wish to define these input variables for general $(N$ dimensional) lattices, for various kind of controls (such as, for instance, distributed or boundary controls) and in such a way that the forced solution exists for any admissible controls. For this, we consider:

- a lattice $\mathcal{T}$, which covers the whole spatial domain of interest;

- a region $\mathcal{T}^{x} \subset \mathcal{T}$ where the lattice sites can be excited;

- a state space $Z_{l b m}$ containing $x(k)$ or $z(k)$ here above

- a control space $\mathcal{U}=l_{2}\left(\mathcal{T}^{x}, \mathbf{R}\right)$ with

$$
l_{2}\left(\mathcal{T}^{x}, \mathbf{R}\right)=\left\{\mathbf{u}: \mathcal{T}^{x} \rightarrow \mathbf{R} / \sum_{x_{j} \in \mathcal{T}^{x}} \mathbf{u}\left(x_{j}\right) * \mathbf{u}\left(x_{j}\right)<\infty\right\}
$$

The space $l_{2}\left(\mathcal{T}^{x}, \mathbf{R}\right)$ may be, in the finite dimensional case (finite number of excitable sites in the lattice), identified with the space $\mathbf{R}^{p}$ where $p:=\operatorname{card}\left(\mathcal{T}^{x}\right)$. The $l_{2}$ convergence is then trivial. We will consider this case in the following.

- an input operator $G: \mathcal{U} \rightarrow Z_{l b m}, \mathbf{u} \rightarrow G \mathbf{u}$. Usually, in LB models, local input maps $g_{x_{j}}$ may be defined describing how the states corresponding to the excited site $x_{j} \in \mathcal{T}^{x}$ are locally controlled. The global input operator may then be defined as

$$
G \mathbf{u}=\left\{\begin{array}{l}
g_{x_{j}}\left(\mathbf{u}\left(x_{j}\right)\right) \text { if } x_{j} \in \mathcal{T}^{x} \\
0 \text { if not }
\end{array}\right.
$$

In the general case, these definitions result in a LBM controlled system

$$
x_{k+1}=\mathcal{F}_{l b m}\left(x_{k}, \mathbf{u}_{k}\right), x_{0} \in Z_{l b m}
$$

In the linear case it reduces to a classical state space system

$$
z_{k+1}=F_{l b m} z_{k}+G_{l b m} \mathbf{u}_{k}, z_{0} \in Z_{l b m}^{r}
$$

$Z_{l b m}^{r}$ is a reduced state space where controlled states have been removed. $F_{l b m}$ is the matrix obtained when the rows corresponding to excited states (considered as inputs) have been removed from the dynamic matrix 


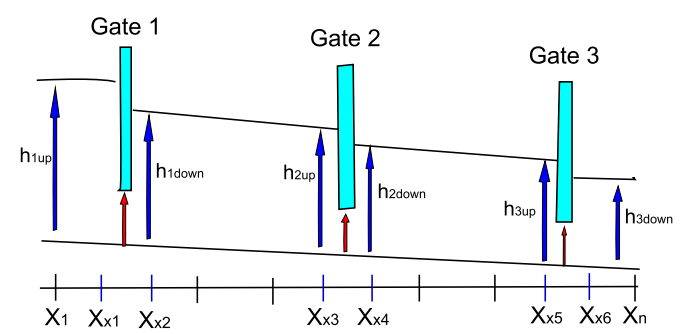

Fig. 3. Interconnected scheme

$T_{f}: F_{l b m}=M^{T} T_{f} M$ where $M$ is the projection matrix corresponding to the cancellation of the states used in the control. $G_{l b m}$ is the control matrix defined as: $G_{l b m}=$ $M^{T} T_{f} M_{c}$, where $M_{c}$ is the projection matrix selecting those columns corresponding to the controlled states. Naturally, $G_{l b m}$ may be viewed as a control operator and $F_{l b m}$ as the infinitesimal generator of a semi-group $\left\{F_{l b m}^{k}\right\}_{k \geq 0}$, Gibson (1972); Packel (1972). In the example of the canal described by the (Fig. 3 ), we assume $n$ sites and 3 gates. We consider the whole canal as the domain $\mathcal{T}$. The two sites around each gate as those belonging to $\mathcal{T}^{x}$. When we introduce a gate in the canal, we can impose some distribution functions to the lattice, see Pham et al. (2010). We can for instance define a control such that:

- if $x_{j} \in \mathcal{T}^{x}$ is before the gate, $\mathbf{u}\left(x_{j}\right)=\varepsilon_{2}\left(x_{j}, k\right)$;

- if $x_{j} \in \mathcal{T}^{x}$ is after the gate, $\mathbf{u}\left(x_{j}\right)=\varepsilon_{1}\left(x_{j}, k\right)$.

And this, for each gate. If the sites before the gates are, for example, $x_{2}, x_{8}$ and $x_{14}$, we shall have as control:

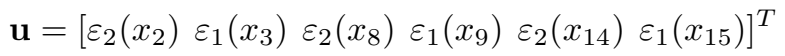

Matrices $F_{l b m}$ and $G_{l b m}$ for this example are easily derived by noticing that the rows to be withdrawn from the dynamic matrix $T_{f}$ for form the matrix $F_{l b m}$ are : 6, $8,24,26,42$ and 44 , and the matrix $M$ is obtained by withdrawing the columns corresponding in the identity matrix.

\section{CONTROLLABILITY FOR LARGE SCALE LB CONTROL SYSTEMS}

\subsection{Analytical or direct methods}

To determine the controllability of a system, either the controllability matrix or the controllability Gramian may be used. For the LB control systems (21), the controllability matrix is given by

$$
\mathcal{C}=\left[\begin{array}{llll}
G_{l b m} & F_{l b m} G_{l b m} & \ldots & F_{l b m}^{m-1} G_{l b m}
\end{array}\right]
$$

where $m$ is the dimension of $F_{l b m}$. The LB control systems is then controllable if this controllability matrix is full row rank. Controllability Gramian defined as $\mathcal{C C}^{T}$ may be computed as

$$
W_{c}=\sum_{\tau=0}^{m-1} F_{l b m}^{\tau} G_{l b m} G_{l b m}^{\prime}\left(F_{l b m}^{\prime}\right)^{\tau}
$$

Equivalently, the LB control systems will be controllable if $W_{c}$ is full rank.

Due to the large scale of the considered LB control system, it is very difficult to obtain any analytical result for the structural behavior of the controllability matrix. In fact we do not even succeed in finding some qualitative structural information about this matrix. Similarly, we neither obtain analytical expressions for the controllability Gramians. Therefore we focused on the numerical solution for controllability Gramians which are (semi)positive solutions of Lyapunov equations:

$$
\left\{\begin{array}{l}
F_{l b m} W_{c} F_{l b m}^{\prime}+G_{l b m} G_{l b m}^{\prime}=W_{c} \\
W_{c} \geq 0
\end{array}\right.
$$

Hence various LMI/LME tools may be tried to compute the controllability Gramians. We tried the Yalmip toolbox for Matlab (see Lofberg (2004)). However this tool is not suited for large scale (sparse) dynamical systems where it provides meaningless values for the controllability Gramians (complex or negative eigenvalues).

The Matlab's toolbox Lyapack may be used to compute the Lyapunov equation solution for larger scale and sparse problems Penzl (2000). Originally it is designed for continuous problems. However the Cayley's transform (see Haynes (1991)) may be used to convert the discrete Lyapunov equation (25) into a continuous Lyapunov equation of the form $A W_{c}+W_{c} A^{T}=D$ with

$$
\left\{\begin{array}{l}
A=\left(F_{l b m}-I\right)\left(F_{l b m}+I\right)^{-1} \\
D=-0.5 *\left(I-F_{l b m}\right) G_{l b m} G_{l b m}^{T}\left(I-F_{l b m}^{T}\right)
\end{array}\right.
$$

However, using this approach (with $n=200$ sites) gives large maximal eigenvalues for the residuals which are respectively 1.33 and $1.59 e+02$ in discrete Lyapunov equation (25) and in the continuous Lyapunov equation obtained from the Cayley transform (26). Therefore, we finally adopted a fixed point iterative method to solve our Gramian computation problem.

\subsection{An iterative method for solving Stein's equation}

Stein's equations are equations of the general form

$$
A X A^{\prime}-X+C=0
$$

Lyapunov's equation is thus a particular case. Since they are fixed point problems, iterative schemes such as Smith's iterations (see Benner et al. (1999))

$$
\begin{aligned}
& X_{0}=C \\
& X_{k+1}=C+A X_{k} A^{\prime} \text { for } k=0,1,2, \ldots
\end{aligned}
$$

may be used to solve numerically the problem. One of the advantages of such an iterative method is that it is perfectly suited to sparse matrix problems. This reduces drastically the complexity for large scale sparse problems like the Gramian computation of LM control systems. Moreover, in our case, the error estimation in Frobenius norm $\|.\|_{F}$

$$
\rho_{k}=\left\|W_{c, k}-W_{c, k-1}\right\|_{F}
$$

rapidly converges to $1.0 e-17$ (the machine accuracy).

From Benner et al. (1999), if $\sigma(A)<1$, there exist real constants $0<L$ and $0<r<1$ such that

$$
\left\|X-X_{k}\right\|_{2} \leq L\|C\|_{2}(1-r)^{-1} r^{2^{k}}
$$

This shows that the method converges for all equations with a Schur stable coefficient matrix $A$. And with this, we 
tried to see how vary the controllability, or how grow the energy to give to the system for make it achieves a wanted state. The results are presented in the next section.

\section{PRESENTATION OF RESULTS}

\subsection{Simulation setup}

We consider the canal from figure 3 discretized with a $n$ sites $D 1 Q 3$ lattice. Numerical values for the canal parameters are those from an experimental plant in the LCIS laboratory in Valence, France. We used the gravitational acceleration $g=9.81 \mathrm{~ms}^{-2}$; the width $B=0.1 \mathrm{~m}$, the length $L=8 m$, the Manning's coefficient $n_{m}=0.01 \mathrm{~s} / \mathrm{m}^{1 / 3}$ and the relaxation time $\tau=1.2 \mathrm{~s}$. The parameters of the model are defined such that $\delta x=L /(n-1)$ and $\delta t=\delta x / 2$. This corresponds to a numerical lattice velocity of 2 which make the method suitable for the subcritical Shallow Water Flows.

\subsection{Threshold of controllability}

To determine controllability, it seems natural to check if the controllability matrix is full row rank. Under the considered configuration, it appears that the system is controllable (under Kalman's criterion) until the number of sites reaches $n=30$. The lost of controllability corresponds to lower eigenvalues of the controllability matrix under the floating point accuracy. It is thus possible in this example to define a numerical threshold of controllability which will correspond to a maximum number of sites. In the next subsections, the effect on the controllability of growing of the number of sites beyond this maximum will be investigated.

\subsection{Energy convergence}

We consider the given system in a zero initial state and will compute the minimum input signal energy needed to reach another state, given by $x_{r}=0.033 *\left[\begin{array}{llll}1 & 1 & \cdots & 1\end{array}\right]^{T}$ with $\operatorname{dim}\left(x_{r}\right)=m$ and $m=3 * n-6$, the dimension of the state space $Z_{l b m}^{r}$. We also consider for simplicity periodic boundary conditions (distributions in the first and last sites are made equals, see Pham et al. (2010)). These boundary conditions allow to compensate the dissipated energy in the canal, injecting it at the upstream end.

We expect that for a controllable LB control system, the state $x_{r}$ may be reached with a control signal with finite energy, whatever the number of sites is. Indeed the model is constructed on invariant macroscopic properties (total mass, momentum, energy) which are indeed invariant quantities in the numerical simulations. However, we observe numerically (see Fig. 4) that the energy grows quickly with the number of sites. The same remark applies for the Gramian trace represented in (Fig. 5). The two previous figures are obtained with Gramians computed with the Smith's iteration algorithm with residual error in the discrete Lyapunov equation (25) near the machine's precision .

\section{CONCLUDING REMARKS}

In this paper we considered LB models for distributed parameters systems. It has been shown how the local

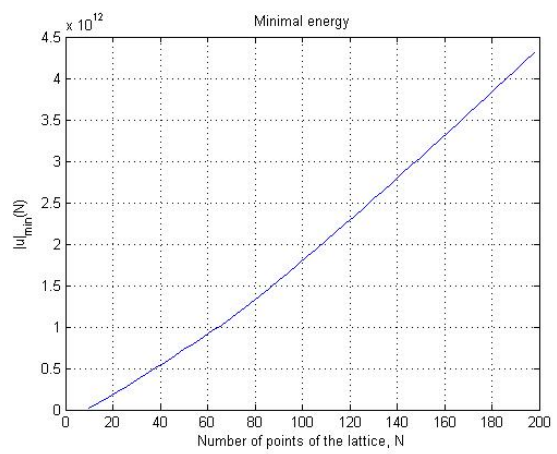

Fig. 4. Energy of system

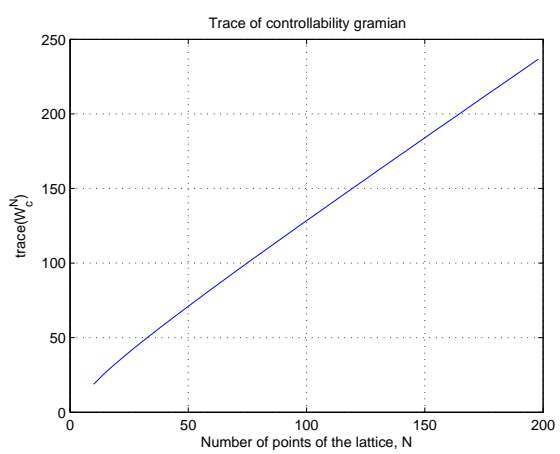

Fig. 5. Trace of Gramian

evolution equation and equilibrium closure equations in LBM may be used to obtain a global state space model for the microscopic distributions.

We shown how a linearization around a macroscopic stationary profile may be derived on the shallow water equations example to derive a linearized state space model for the distributions small variations.

We also shown on this shallow water example how input variables may be introduced to derive a LBM control system similar to classical finite dimensional approximations of boundary control systems.

We also shown that the derived LB control system numerically looses its controllability property when the number of sites increases beyond a limit. Neither the minimum energy to reach a given state, nor the trace of the controllability Gramian seem to be bounded when this number of sites increases.

These numerical results clearly invite us to a deeper analysis and understanding of what numerical convergence of the LB models (which has been proven to be quadratic in space and time for the state, Zhou (2004)) means in terms of controllability or observability properties. More precisely, it appears that the meaning of controllability itself has to be restricted in an appropriate way if we expect convergence results. From one side, one has to consider energy supplied through the inputs for clearly identified scenarii and from the other side it seems that local (regional) controllability property has to be considered with respect to some controllability numerical threshold. 


\section{REFERENCES}

Benner, P., Quintana-Orti, E.S., and Quintana-Orti, G. (1999). Solving Stable Stein equations on distributed memory computers. Lecture Notes in Computer Science, Volume 1685/1999, 1120-1123.

Bhatnagar, P., Gross, E., and Krook, M. (1954). A model for Collision Processes in Gases. i. Small Amplitude processes in Charged and Neutral One-Component Systems. Physical Review, 94(3), 511-525.

Chopard, B. and Droz, M. (2005). Cellular Automata Modeling of Physical Systems. Cambridge University Press.

Chopard, B. and Lagrava, D. (2006). A cellular automata model for species competition and evolution. In Proc. of the Int. Conf. on Cellular Automata for Research and Industry ACRI'06. Perpignan, France.

Chopard, B., Ouared, R., Deutsch, A., Hatzikirou, H., and Wolf-Gladrow, D. (2010). Lattice-Gas Cellular Automaton Models for Biology: From Fluids to Cells. Acta Biotheoretica, 1-12. DOI 10.1007/s10441-0109118-5.

Gibson, A.G. (1972). A discrete Hille-Yosida-Phillips Theorem. Journal of Mathematical Analysis and Applications, 39, 761-770.

Haynes, T. (1991). Stable matrices, the Cayley transform, and convergent matrices. Internat. J. Math. \& Math. Sci., 14, 77-82.

Hencinas, L.H., White, S.H., del Rey, A.M., and Sanchez, G.R. (2007). Modelling forest fire spread using hexagonal cellular automata. Applied mathematical modelling, $31(6), 1213-1227$.

Lofberg, J. (2004). Yalmip: A Toolbox for Modeling and Optimization in Matlab. Computer Aided Control Systems Design, 2004 IEEE International Symposium, 284-289.

Neuman, J.V. (1966). The theory of self-reproducing automata. University of Illinois Press.

Ostfeld, R., Glass, G., and Keesing, F. (2005). Spatial epidemiology: an emerging (or re-emerging) discipline. Trends in Ecology and Evolution, 20(6), 328-336.

Ouared, R., Chopard, B., and Rufenacht, D. (2006). A Lattice Boltzmann simulation of clotting in stented aneursysms and comparison with velocity or shear rate reductions. Mathematics and Computers in Simulation, 72(2-6), 108-112.

Packel, E.W. (1972). A Simplification of Gibson's Theorem on Discrete Operator Semigroups. Journal of Mathematical Analysis and Applications, 39, 586-589.

Penzl, T. (2000). A Matlab Toolbox for large Lyapunov and Riccati equations, Model reductions problems, and linear-quadratic optimal control problems.

Pham, V.T., Chopard, B., Lefèvre, L., Anda Ondo, D., and Mendes, E. (2010). Study of the 1d Lattice Boltzmann shallow water equation and its coupling to build a canal network. Journal of Computational Physics, 229, 73737400 .

Salmon, R. (1999). The lattice Boltzmann method as a basis for ocean circulation modeling. J. Marine Resarch, 57, 503-535.

Slimi, R., Yacoubi, S.E., Dumonteil, E., and Gourbire, S. (2009). A cellular automata model for Chagas disease. Applied mathematical modelling, 33(2), 1072-1085.
Zhou, J.G. (2004). Lattice Boltzmann Methods for Shallow Water Flows. Springer.

\section{Appendix A. MATRICES}

In the sequel we will use the notations $F r^{2}=\frac{u^{2}}{g h}$ and $\Psi^{2}=$ $\frac{v^{2}}{g h}$ to denote respectively the real Froude number and a kind of lattice Froude number. The real Froude number is an a-dimensional parameter characterizing locally the kind of flow arising at the considered location. It expresses the quotient between the kinetic and potential energies. $F r<1$ corresponds two a "fluvial" flow, $F r>1$ represents a "torrential" flow and $F r=1$ corresponds to a critical flow.

$$
\begin{aligned}
& A_{0}=\frac{1}{\tau}\left[\tau-\frac{1}{2 \Psi^{2}} 1-\frac{1}{2 \Psi^{2}}-\frac{F_{r}}{\Psi} 1-\frac{1}{2 \Psi^{2}}+\frac{F_{r}}{\Psi}\right] \\
& A_{1}=\frac{1}{\tau}\left[\frac{1}{4 \Psi^{2}} \frac{1}{4 \Psi^{2}}+\frac{F_{r}}{2 \Psi}+\tau-\frac{1}{2} \frac{1}{4 \Psi^{2}}-\frac{F_{r}}{2 \Psi}-\frac{1}{2}\right] \\
& A_{2}=\frac{1}{\tau}\left[\frac{1}{4 \Psi^{2}} \frac{1}{4 \Psi^{2}}+\frac{F_{r}}{2 \Psi}-\frac{1}{2} \frac{1}{4 \Psi^{2}}-\frac{F_{r}}{2 \Psi}+\tau-\frac{1}{2}\right]
\end{aligned}
$$

and by letting $a=\frac{1}{\Psi^{2}}\left(1-F r_{e}^{2}\right)$ and $b=I+J_{e}+\frac{4 J_{e} R_{e}}{3 h_{e}}$, we have

$$
\begin{gathered}
T_{0}=\frac{1}{\tau}\left[\begin{array}{llll}
\tau-a & 1-a-2 \frac{F r_{e}}{\Psi_{e}} & 1-a+2 \frac{F r_{e}}{\Psi_{e}}
\end{array}\right] \\
T_{1}=\frac{1}{\tau}\left[\begin{array}{llll}
\frac{a}{2} & \tau-\frac{1}{2}+\frac{a}{2}+\frac{F_{e}}{\Psi_{e}} & \frac{a}{2}-\frac{1}{2}-\frac{r_{e}}{\Psi_{e}}
\end{array}\right] \\
T_{2}=\frac{1}{\tau}\left[\begin{array}{lllll}
\frac{a}{2} & \frac{a}{2}-\frac{1}{2}+\frac{F r_{e}}{\Psi_{e}} & \tau-\frac{1}{2}+\frac{a}{2}-\frac{F r_{e}}{\Psi_{e}}
\end{array}\right] \\
N=\frac{\delta t}{2 v} g\left[\begin{array}{lllll}
b & \left(b-\frac{2 J_{e} v}{u_{e}}\right) & \left(b+\frac{2 J_{e} v}{u_{e}}\right)
\end{array}\right] \\
A=\left[\begin{array}{cccccc}
A_{0} & 0 & 0 & \cdots & 0 & 0 \\
0 & 0 & 0 & \cdots & 0 & A_{1} \\
0 & A_{2} & 0 & \cdots & 0 & 0 \\
0 & A_{0} & 0 & \cdots & 0 & 0 \\
A_{1} & 0 & 0 & \cdots & 0 & 0 \\
0 & 0 & A_{2} & \cdots & 0 & 0 \\
& & & \vdots & \\
0 & 0 & 0 & \cdots & A_{0} & 0 \\
0 & 0 & 0 & \cdots & 0 & 0 \\
0 & 0 & 0 & \cdots & 0 & A_{2} \\
0 & 0 & 0 & \cdots & 0 & A_{0} \\
0 & 0 & 0 & \cdots & A_{1} & 0 \\
A_{2} & 0 & 0 & \cdots & 0 & 0
\end{array}\right]
\end{gathered}
$$

The matrix $T$ is obtained by replacing in the matrix $A$ each matrix $A_{i}$ by a matrix $T_{i}$ with the same direction $i$.

$$
T_{f}=\left[\begin{array}{cccccc}
T_{0} & 0 & 0 & \cdots & 0 & 0 \\
0 & 0 & 0 & \cdots & 0 & T_{1}+N \\
0 & T_{2}-N & 0 & \cdots & 0 & 0 \\
0 & T_{0} & 0 & \cdots & 0 & 0 \\
T_{1}+N & 0 & 0 & \cdots & 0 & 0 \\
0 & 0 & T_{2}-N & \cdots & 0 & 0 \\
& & & \vdots & & \\
0 & 0 & 0 & \cdots & T_{0} & 0 \\
0 & 0 & 0 & \cdots & 0 & 0 \\
0 & 0 & 0 & \cdots & 0 & T_{2}-N \\
0 & 0 & 0 & \cdots & 0 & T_{0} \\
0 & 0 & 0 & \cdots & T_{1}+N & 0 \\
T_{2}-N & 0 & 0 & \cdots & 0 & 0
\end{array}\right]
$$

